Journal of Engineering and Applied Sciences 14 (20): 7736-7742, 2019

ISSN: 1816-949X

(C) Medwell Journals, 2019

\title{
Design Sensitivity Analysis and Performance Optimization of Axial Flow Pump
}

\author{
${ }^{1}$ Malik N. Hawas, ${ }^{2}$ Aya Adnan Yaseen and ${ }^{3}$ Akeel A. Mohammed \\ ${ }^{1,3} \mathrm{Al}$-Furat Al-Awsat Technical University, Al-Najaf, Iraq \\ ${ }^{2}$ Al Mansour University College, Baghdad, Iraq
}

\begin{abstract}
In modern life, pumps are attached to a huge amount of applications, therefore, some replacement parts should modified and produce in form to be suitable for different configurations. Working with these modification and constraints lead to redesign the geometry to avoid the cavitation phenomena and make the pumps parts as portable as easy to replace at any time. In complex fluid domain like centrifugal pump, there is some sensitivity towards many design parameters and also to some particular objective functions. Investigate and determine the main parameters which have a major effects on the pump performance and efficiency are very important before any further steps. These parameters include contact pressure, Mises stresses, specific speed, coefficient of friction, casing thickness value and clearance between rotor vanes and casing. In this research, the commercial software (Abaqus/CAE) has been adopted as a powerful tool for modelling and simulation this analysis process. Furthermore, the global sensitivity analysis are used to validate and expand the results. Here, some design variables are used to be fixed, like phase angle and angle of blades. In this global sensitivity process, many and different models are built to facilitate the analysis. Meanwhile, in this research, the interactions between some impacts which effects on performances of impellers are resolved. It's found that, the design parameters that has most effecting in reducing stresses can be identifies through sensitivity analysis. Results revealed that, the contact pressure, Mises stresses and the shape parameter ( $\mathrm{t}$ ) are the most effecting parameters. Simulation results show that, there's a positive relationship between specific speed and efficiency. Furthermore, it's found that the blade angle influenceon vane performances can be neglected comparing with other design variables. The finding of optimization analysis shows that, the efficiency of optimized model is higher than the conventional type. It's also concluded that the overall performances will enhance by this type of optimization process. The minimum pressure coefficient and efficiency can improve by $(0.35$ and $0.4 \%)$, respectively.
\end{abstract}

$\underline{\text { Key words: Axial pump, efficiency, simulation, sensitivity and optimization, design parameters, blade angle }}$

\section{INTRODUCTION}

The axial flow pump (propeller pump) is one kinds of centrifugal pump which used vanes to direct the flow axially and differs from the other types that direct the flow radially. Pressure head in axial flow pumps normally less than radial types but the flow rates can be much higher. The pump impeller in this type is typically consisting of three to four vanes (Blaszczyk et al., 2016).

The flow rate produced by this type of pumps is considering the highest value from the other centrifugal pump types while the generated head is lower than other types (Li et al., 2013). Performance characteristics of axial flow pumps are different from many other types. Even though their pressure head is low but they have much capacity comparing with other similar centrifugal types. Unlike the radial flow pumps when the flow rates decreased the required horsepower increase (Jones, 2014). This type of pumps are normally used in some applications that's requires low head and high flow rates. It's useful for circulating large amounts and different types of fluids in power plants, chemical industry, waste water, irrigation and flood dewatering (Derakhshan et al., 2013).

Low-lift surface water and efficiency can improve significantly by using the axial flow pumps. Furthermore, energy consumption and water discharge will also improve. In this type, water is lifted due to the negative pressure created by the centrifugal force made by the impeller. The main working principle of this pump includes that the rotating impeller will generate high centrifugal force and negative pressure which cause the water lifting due this pressure (Valle et al., 2014). 
Selecting the requirement conditions of flow rate and head is the first design step of the axial pump, also the value of specific speed in axial flow pump will establish the impeller type. There are some considerations are governed on speed selection like specific speed, driver type and optimum efficiency.

At this time being, using modelling and simulation in many industrial engineering process can assist in design optimisation and minimize the experimental and computational costs. These surrogate models can mimic the empirical test and leads to important forecasting results in short time comparing with practical (Xu et al., 2018). Design parameters which has main effects on centrifugal pump performance and the optimization technique effectiveness have been discussed (Siddique et al., 2018).

Now a days, simulation and optimization process are widely used in many industrial application to enrich the results and minimize the percentage of error before experimental part. Finite element Software (Abaqus) is a powerful tool and widely used for evaluating and developing such models to determine and estimate the parameters that have the main effects on pump configurations (Chimenti et al., 2004).

Results of simulation process are very essential, especially by using Finite Element Analysis (FEA) which consider an important step before any further action. Shape and topology optimization is important to reduce the critical zones which recirculation occurs. Setup any optimization related to pump efficiency should be emphasis the outlet velocity and minimizes the values of pressure drop.

Normally, axial flow pump has a significant reduction in scale of the axial geometry with high impact in energy and better cavitation performances comparing with other types (Yadav et al., 2016; Bhaoyal et al., 2010). Optimization process is generally used by designer engineer as a power tool to generate a new design for optimizing parts. This tool will help for investigate and increase the understanding of the nature and dynamic flow for further solutions like, casing geometry, vanned diffuser profile and shape of blade profile (Kim et al., 2010; Walker and Shelley, 2010).

\section{MATERIALS AND METHODS}

Design process and model development: A pump selection is normally depends on the relation between discharge and head. Procedure of pump design is following some important steeps like case design and impeller design. Setup and measurement the output flow

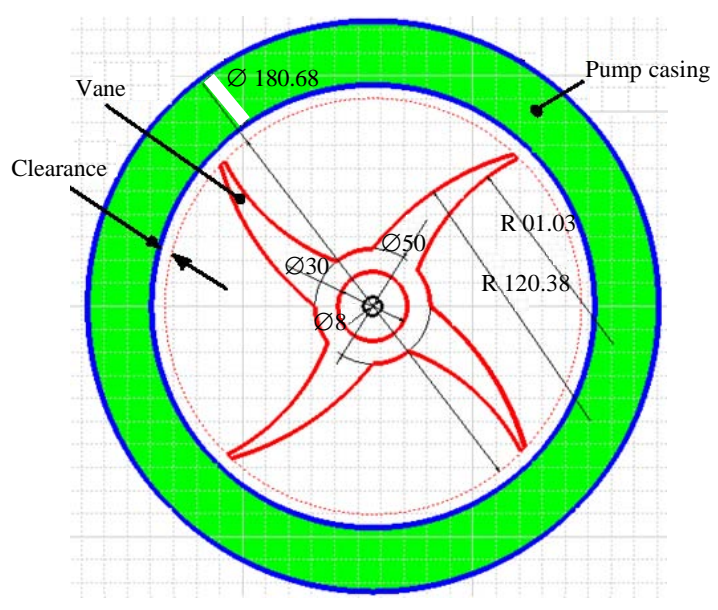

Fig. 1: Geometry of two dimensional vanes

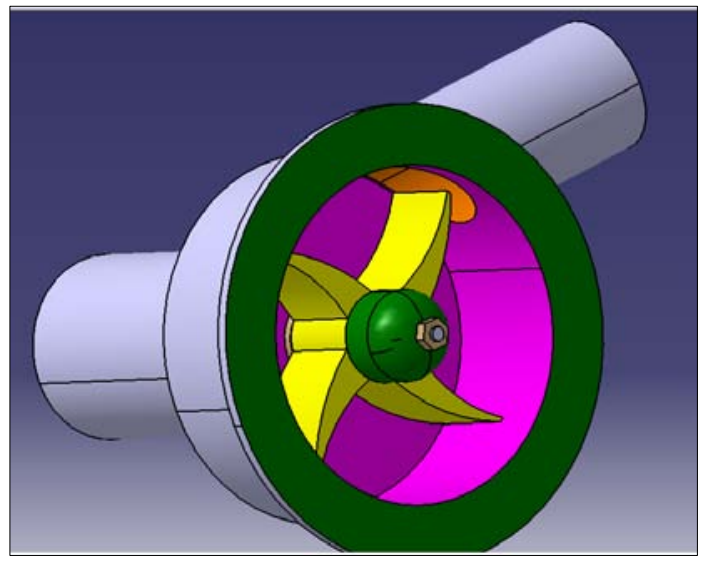

Fig. 2: Geometry of pump casing in three dimensions

is required to estimate the flow parameter related to the interaction between impeller and pump casing. Geometry of rotating vanes and pump casing in two and three dimension including the important dimensions are shown in Fig. 1 and 2, respectively.

The conventional active parts in axial pump assembly model are consisting from vanes, propeller and vane casing. Figure 3 represent across section for illustrating these parts. Pumping performance is considered and refers to dynamic performance of blade and casing. Interaction between pump blades and fluid inside casing in addition to the clearance between vane and casing are considered as design critical element. This interaction can give insight used in optimization process for this design before any further analysis steps.

Simulation and numerical analysis: $\mathrm{Abaqus} / \mathrm{CAE}$ has been employed as finite element software for analysis 


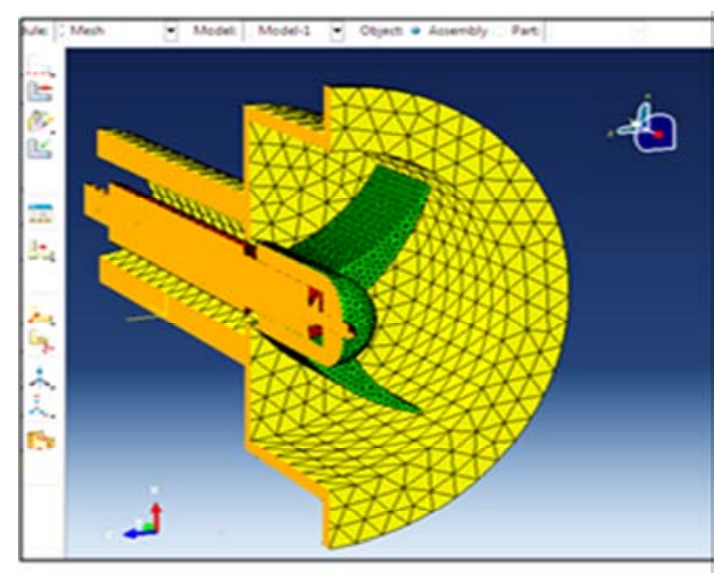

Fig. 3: Assembly cross section in (3D)

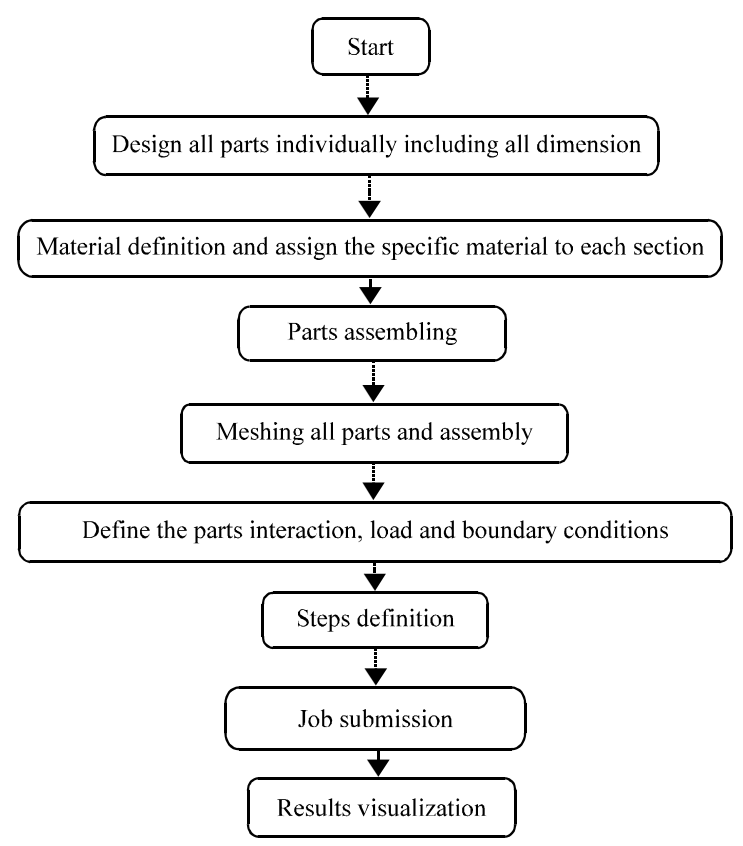

Fig. 4: Simulation procedure by Abaqus Software

and simulation process in this research paper. Figure 4 illustrate the simulation procedure by this software.

This designed model has been restricted to involve vanes and vane casing. In boundary condition, the pump casing is considered as a non-deformable body (rigid body). Therefore, it may fixed and constrain from any movement in all directions. The interaction between pump vanes and pump casing are modelled by using Coulomb friction coefficient of (0.2) with finite sliding contact.

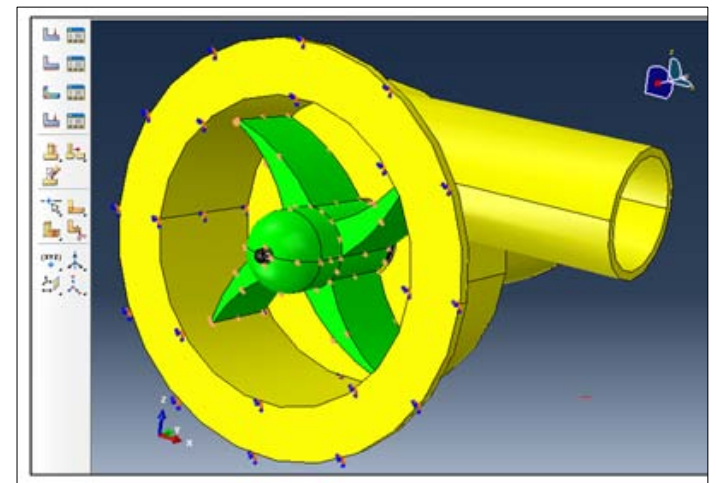

Fig. 5: Illustrate this boundary condition

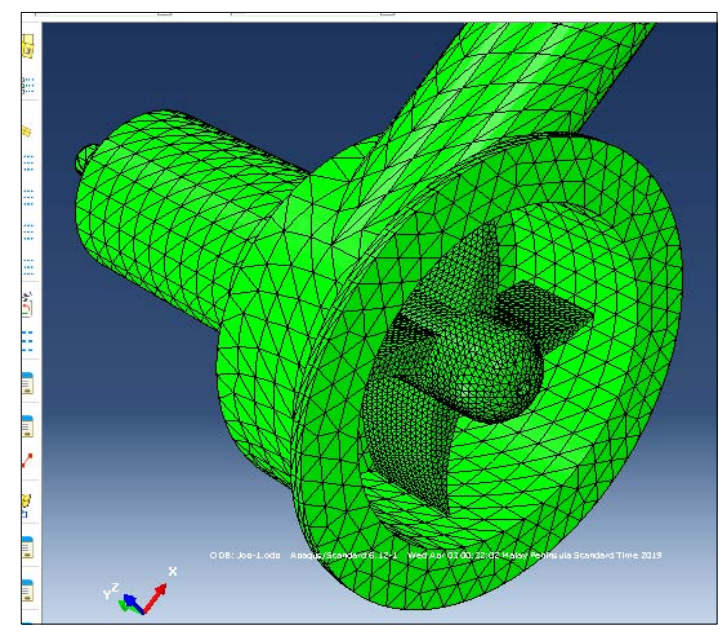

Fig. 6: Illustrate the generated mesh the assembled parts

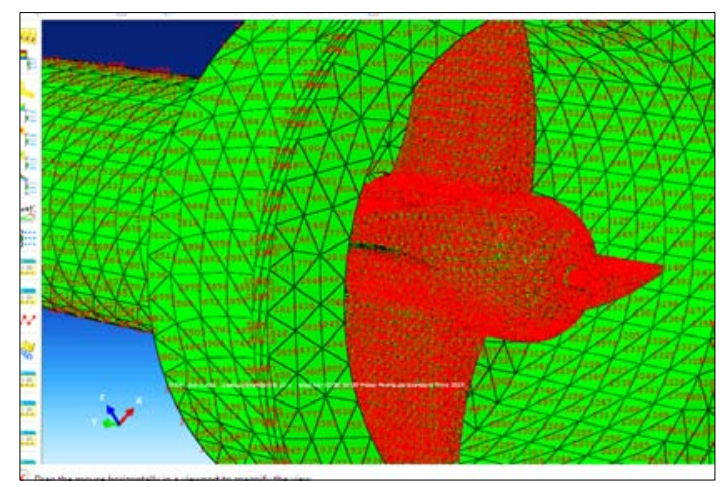

Fig. 7: Elements label for whole model

Design parameters in this study are shape parameters and coefficient of friction (0.2) which is used to find the friction effects on the interacting faces. Figure 5 illustrate this boundary condition (Fig. 6 and 7). 
Shape parameters are important in study the effects of aspect of housing geometry on pump stiffness. These parameters are includes housing thickness ( $t$ ), clearance between vane tip and inside casing peripheral (c) and inside housing diameter (d). Parameter shape variation option is adopted to find the shape gradients during optimization process.

In any accurate simulation, meshing all parts individually is very important before any further analysis step. Bottom-up meshing technique is used for all parts after seeding each part separately by using the approximation global size (3.7) and curvature deviation factor $(0.1)$.

In mesh controls, (Hex-dominated) with bottom-up technique are used to in controlling the generated element. The approximation number of element are (8) elements per each circle. Figure 6 illustrate the generated mesh for all assembled parts.

The option of determine the whole elements in all model is very essential in these types of analysis. However, counting and locating the numbers of elements and nodes labels for this model will facilitate all visualization results at any node or element exactly. Elements label for whole model are illustrate in Fig. 7.

\section{RESULTS AND DISCUSSION}

In this simulation, the pump casing has been restricted as mentioned in description above. That's means casing is a fixed part in horizontal direction and all related nodes has been constrained in all directions. The motion of rotating vanes is simulated, according to the reference point of the main rotation axis. The Mises stress sensitivity is taken as primary response in model design. Also, the contact pressure behaviour between fluid with both vanes and casing should take in to consideration. Contour of Mises stress in Fig. 8 show the distribution of these stresses and their effects on blade configuration. It's obvious from the contour of von-Mises stresses that the magnitude and concentration of stress distribution is much higher in the lower part of existing stream than the upper side due to the effects of fluid friction on the entire case.

It's found that the contact pressure is highly sensitive to thickness value. That's means decreasing in thickness will decrease the contact pressure while decrease the clearance will increase the contact pressure. It's advised for positive changing between the thickness and contact pressure. Figure 9 show the distribution of contact pressure around axil of suction zone.

Various combinations of design sensitivity analysis in this model are considered in dynamically loaded over the pump vanes. Also, the analysis formulation stiffness matrix are compared to find the maximum sensitivities values. Plane strain model with (670) elements are use in modelling of pump casing. Also polynomial strain energy with incompressible hyperplastic material is used to simulate the behaviour of impeller vanes. Table 1 is listed the values of normalized sensitivities for the highest values of the von-Mises stresses in the lower zone of existing part.

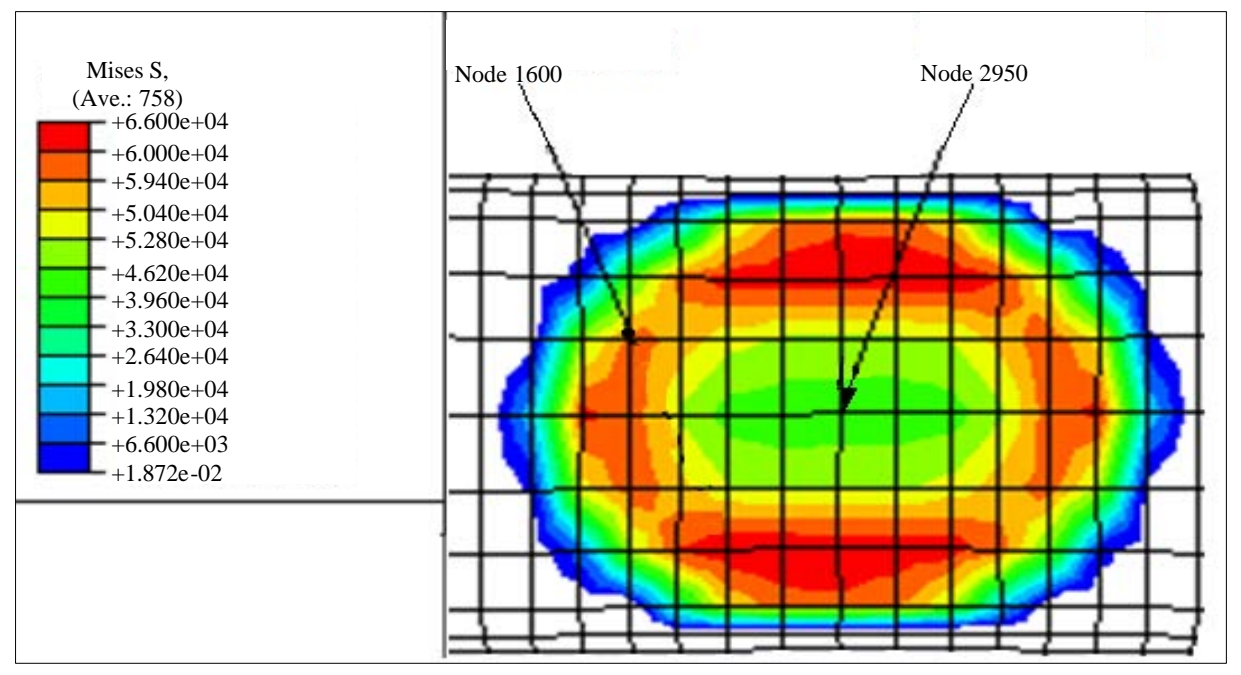

Fig. 8: Distributionand values of von-Mises stresses 


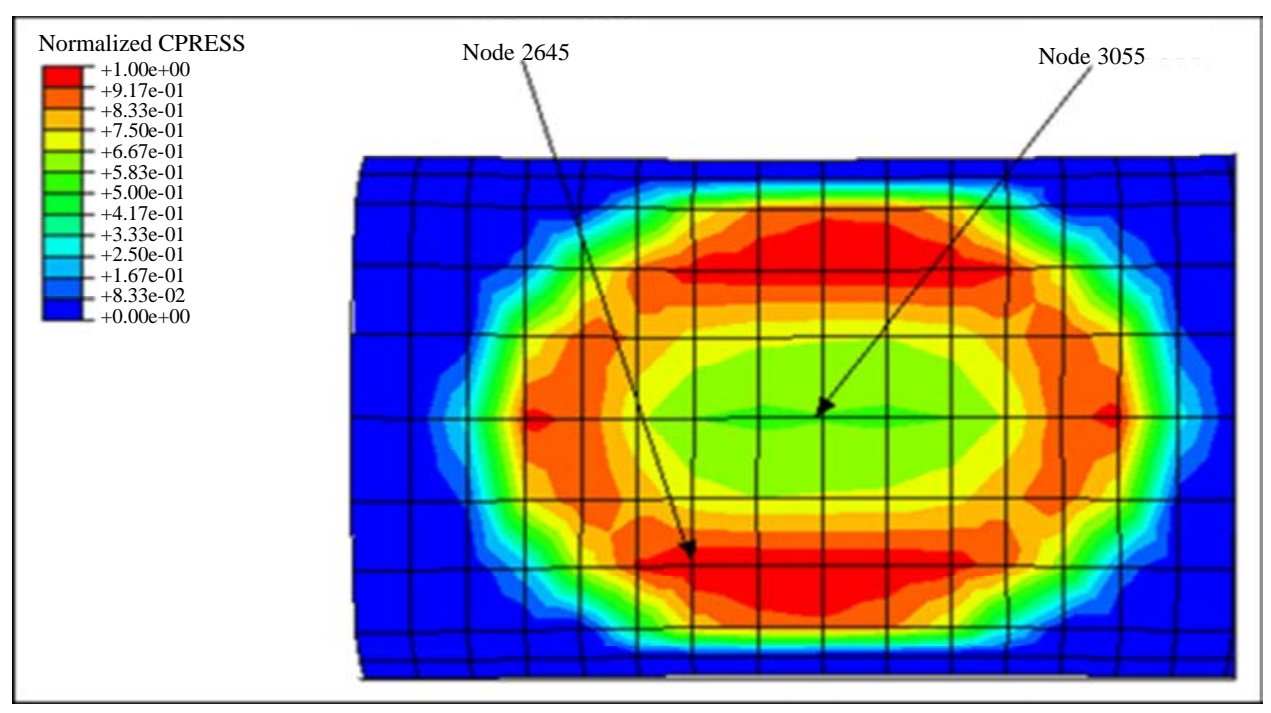

Fig. 9: Distribution of contact pressure around axil of suction zone

Table 1: Percentages sensitivity values in both contact pressure and Mises-stress

\begin{tabular}{lcc}
\hline & Total incremental sensitivity values \\
\cline { 2 - 3 } & von-Mises stress (\%) & Contact pressure (\%) \\
\hline $\begin{array}{l}\text { Design parameters } \\
\text { tip and casing }\end{array}$ & 0.31 & 0.24 \\
Contact Pressure (P) & 1.12 & \\
Clearance value & 1.95 & 0.12 \\
Blade thickness (t) & 1.70 & 0.45 \\
Coefficient of friction & 0.10 & 0.88 \\
\hline
\end{tabular}

It's concluded from Table 1 that the von-Mises stresses is more sensitive to the thickness than other dimensions and coefficient of friction. Also, it's concluding that, if we want to reduce the stresses consideration, the thickness must be considered. An $(18 \%)$ reduction in thickness will leads to an $(8 \%)$ reduction in Mises stresses. Percentage of predicted reduction in the Mises stress is about [5.75 $0.225 \times 0.2+0.101 \times 0.1]$. In such simulation process; results accuracy and the total estimated efficiency are widely depend on mesh accuracy and refinement. There's a strong relation between mesh accuracy and efficiency whereas the efficiency will increase regarding to mesh accuracy as shown in Fig. 10.

Specific speed (Ns) in axial flow pump is likely an index normally used to characterize and predict the pump performance and efficiency. According to this analysis, and referring to the relation between the discharge and pump net positive section head, it's found that the relation between specific speed and efficiency is likely as in Fig. 11. For compaction purposes between conventional axial flow pump and this simulation results in term of horse power rating vise the discharge, it's found that the results of simulation is revealed a better records than conventional as illustrated in Fig. 12.

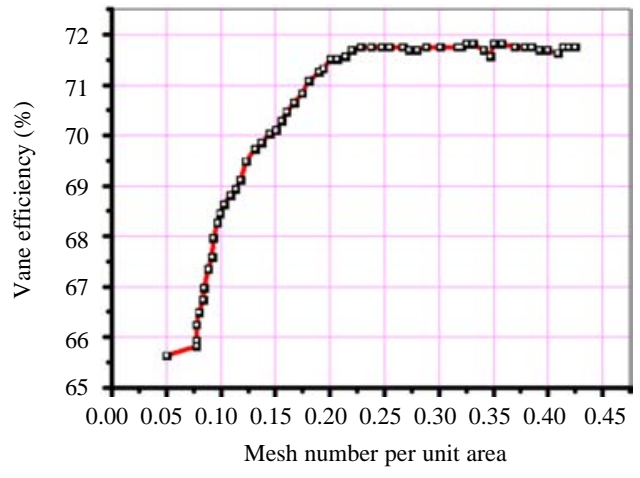

Fig. 10: Relation between mesh accuracy and efficiency

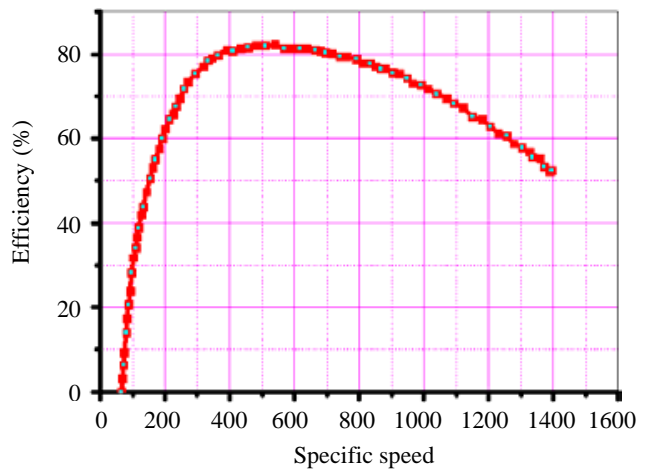

Fig. 11: Illustrates the relation between specific speed and efficiency

Sensitivity analysis results: Normalizing the sensitivities is used by dividing their values on higher values of Mises stress and then scaling the output values by the primary values that use in design parameter. Shape parameters has 


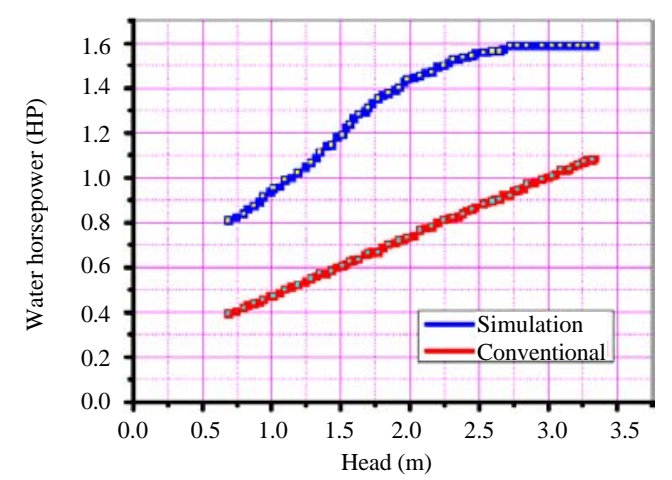

Fig. 12: Compaction between conventional and simulation in HP rating

Table 2: Results of new sensitivity values

\begin{tabular}{lc}
\hline Design parameters & New sensitivity values \\
\hline Thickness $(\mathrm{t})$ & 0.300 \\
Contact Pressure $(\mathrm{P})$ & 0.002 \\
Clearance value & 0.500 \\
\hline
\end{tabular}

a major influence on the stress values where these stresses will increased according to, parameters increase and vice versa while the coefficient of friction has an inverse influence on the Mises stresses because it will increase the shearing forces. Also, reducing the wall thickness ( $\mathrm{t}$ ) will leads to reduce the stress concentration. Table 2 show the values of normalizing sensitivities.

Any changes in proposed design will influence by some parameters like the contact pressure and the clearance between rotor vanes and casing and then results of sensitivity values will use to quantify these changes. To determine the error percentage in such approximation, it's better to compare the finding of various compositions to the total finite difference.

However, the von-Mises stress sensitivity results and the controlling shape parameter (thickness) are in good harmony with overall analysis. In some times and if we neglect the unsymmetrical case in this simulation, the design sensitivity analysis may give a non-accurate results for the sensitivities relative to the coefficient of friction. Maximum stresses sensitivities in the structures are less than interaction pressures sensitivities, also sensitivities for less significant parameters are highly influenced by approximations than others dominant sensitivities.

Optimization analysis: In many industrial applications related to fluid dynamic, software tools are used for optimization purposes. Optimization setup is used to decrease the intensity of backflow and increase the velocity value by meshing the impeller plane uniformity. Optimization process includes eliminate back flow,

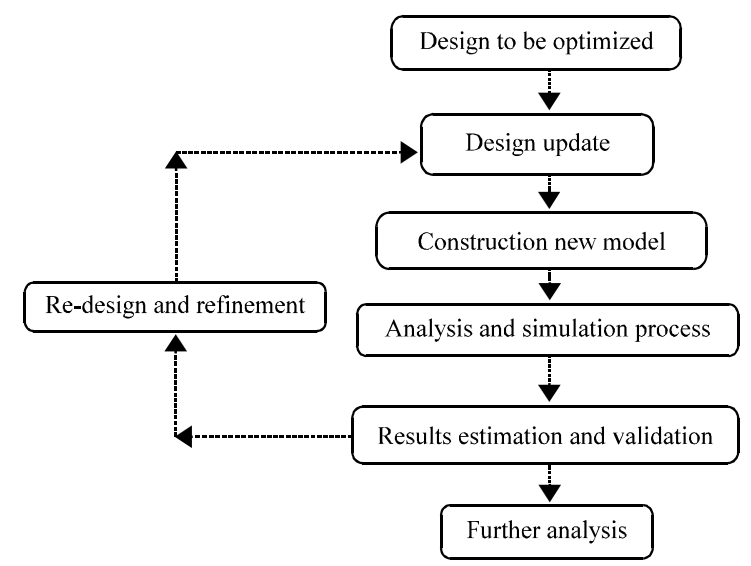

Fig. 13: Optimization flow chart

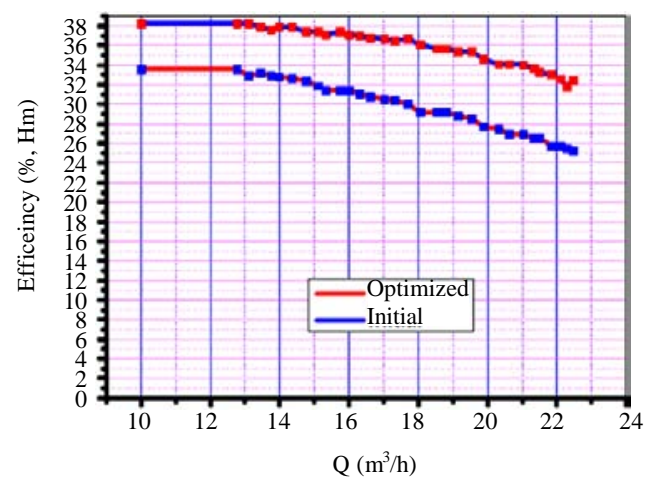

Fig. 14: Comparison between initial efficiency and optimized efficiency

reducing the pressure drop and fluid flow jwill homogeneously distributed. Optimization steps are listed as a flow chart in Fig. 13.

The suitable design and optimization of pump is specified as a combination of influencing factors and requirements on the pump performance. Such factors like pressure, average required discharge, direction of flow, power and material cost. Some significant results are reviled from running this optimized analysis after some changing in the proposed design. It's observed that the percentage of $(12 \%)$ reduction in stress concentration as illustrated in Table 2.

The main aim and objective of optimization isto improve the efficiency. To assure whether the efficiency increase or not all independent variables must be check. For this purpose, any combinations of variables can give high influence on efficiency should be selected. In this case, all independent variables have to be varied and identified. Figure 14 show that a comparison between initial efficiency and optimized efficiency. Whatever, the proposed values and the results output confirm that, the new values will make the model more robust. 


\section{CONCLUSION}

This analysis revealed many significant conclusions. One of the important hints is about the stress concentration which cause degrades the life cycle and performance in both vane propeller and casing. Therefore, it should be reduce to the minimum values. Also, many others conclusions are found and can listed below. Any increase in discharge diameter will lead to minimize the intensity of velocity of outlet jet.

It's concluded that, reducing the stress concentration to the minimum will cause some effects on the whole performance of delivery system. It's found that the main difference between the actual and predicted results is due to nonlinear dependence between design parameter and design response. In many fluid dynamic applications optimization process are built and aim to enhance the understanding of the physics of local fluid, reduce time of development with quality improvement. To optimize the geometry, technical construction and performance, for this type of pumps, further research are recommended.

\section{REFERENCES}

Bhaoyal, B.C., S. Gajbhiye, S. Magdum and S. Kharat, 2010. Weight optimization of split case pump. Simul. Driven Innovation, 1: 1-5.

Blaszczyk, A., A. Papierski and M. Susik, 2016. New design method for the formed suction intake in axial-flow pumps with a vertical axis. Mech. Mech. Eng., 20: 553-568.

Chimenti, M., L. Varela, E.D. Forteza and R. Favaloro, 2004. Computational fluid dynamics analysis of a novel axial flow blood pump with two counter-rotating impellers. Mecanica Computacional, 23: 1483-1489.
Derakhshan, S., M. Pourmahdavi, E. Abdolahnejad, A. Reihani and A. Ojaghi, 2013. Numerical shape optimization of a centrifugal pump impeller using artificial bee colony algorithm. Comput. Fluids, 81: 145-151.

Jones, P., 2014. Design research methods in systematic design. Proceedings of the 3rd International Symposium on Relating Systems Thinking and Design (RSD3), October 15-17, 2014, Oslo, Norway, Europe, pp: 1-7.

Kim, J.H., J.H. Choi, A. Husain and K.Y. Kim, 2010. Performance enhancement of axial fan blade through multi-objective optimization techniques. J. Mech. Sci. Techno., 24: 2059-2066.

Li, D.H., Y. Zhao and G.Y. Wang, 2013. Global design optimization for an axial-flow tandem pump based on surrogate method. IOP. Conf. Ser. Mater. Sci. Eng., 52: 1-10.

Siddique, M.H., A. Afzal and A. Samad, 2018. Design optimization of the centrifugal pumps via low Fidelity models. Math. Prob. Eng., 2018: 1-14.

Valle, S.S., A.S. Qureshi, S. Islam, A. Hossain and M.K. Gathala et al., 2014. Axial flow pumps can reduce energy use and costs for low-lift surface water irrigation in Bangladesh. Master Thesis, CIMMYT, Dhaka, Bangladesh.

Walker, S.W. and M.J. Shelley, 2010. Shape optimization of peristaltic pumping. J. Comput. Phys., 229: 1260-1291.

Xu, S., S. Zhong and H. Zhao, 2018. Optimization of pump hydraulic performance based on the response surface method. Mech. Eng., 1: 1-10.

Yadav, K.K., K. Mendiratta and V.K. Gahlot, 2016. Optimization of the design of radial flow pump impeller though CFD analysis. Intl. J. Res. Eng. Technol., 5: 157-161. 